\title{
Neural auditory (dis)integration underpins phoneme change detection in
}

\section{Wernicke's Aphasia}

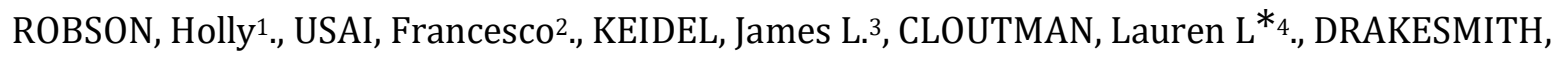
Mark5., SAGE, Karen6., WELBOURNE, Stephen ${ }^{4}$

1: School of Psychology and Clinical Language Sciences, University of Reading, UK

2: Department of Psychology and Neuroscience, Dalhousie University, Halifax (NS), Canada

3: School of Psychology, University of Sussex, UK

4: Sparx, Exeter, UK

5: Cardiff University brain Research Imaging Centre (CUBRIC), Cardiff University, UK

6: Centre for Health and Social Care Research, Faculty of Health and Wellbeing, Sheffield Hallam University, UK

* Corresponding author: Holly Robson h.v.robson@reading.ac.uk, School of Psychology and Clinical Language Sciences, University of Reading, Reading, UK, RG6 6AL

Keywords: Speech perception, categorical perception, EEG, mismatch negativity, power, aphasia, stroke

Acknowledgements: This work was supported by a The Stroke Association, UK (SRTF 2012/02). We would like to thank all the participants and their carers for their involvement in the study. 


\section{$\underline{\text { Abstract }}$}

Speech perception impairments are a universal feature of Wernicke's aphasia (WA) and are systematically related to the Wernicke's-type language comprehension impairment. However, speech perception is not absent; phonological changes can be identified with sufficient acoustic difference between stimuli. This study used a measure of non-instantaneous oscillatory power to explore the neural mechanism associated with impaired and accurate phoneme change detection. Method: Electroencephalography was measured during a multiple deviant mismatch negativity (MMN) paradigm using consonant-vowel-consonant nonword stimuli in participants with WA and neurotypical participants. Oddball stimuli consisted of phonemic changes that could and could not be behaviourally perceived. Sensor level non-zero phase lagged (NZPL) power change, a measure of activity associated with neural interactions, was analysed over the MMN window. Results: Perceptible and non-perceptible phoneme changes were distinguished by NZPL power. Neurotypical and WA participants displayed increased NZPL power to perceptible phoneme changes. Non-perceptible phoneme changes resulted in limited NZPL power change in the neurotypical participants and an unexpected decreased in NZPL power over anterior and central midline and right hemisphere regions in the WA participants. Conclusions: The behavioural percept of phoneme change is associated with wide-spread integrated neural responses. Reduced NZPL power to non-perceived phoneme changes in the WA group indicates reduced neural integration in response to novel phonological information. It is proposed that disrupted ability to form integrated neural responses to auditoryphonological information is a biomarker of the language comprehension impairment in WA. 


\section{$\underline{\text { Introduction }}$}

Acquired language comprehension impairments which occur secondary to left superior temporal lobe lesions are hypothesised to be associated with failures in discrimination and/or identification of speech sounds (Luria, 1976), a condition most commonly referred to as Wernicke's aphasia (WA). When WA persists into the chronic phase, a well-established and systematic relationship can be observed between phonological processing abilities and comprehension (Robson, Pilkington, Evans, DeLuca, \& Keidel, 2017; Robson, Sage, \& Lambon Ralph, 2012). However, phoneme perception abilities are not absent - i.e. accurate discrimination can be made when acoustic differences are sufficiently large (Robson, Keidel, Lambon Ralph, \& Sage, 2012). While acknowledging that the capacity to perform a discrimination task is not equivalent to normal cognitive or neural processing, these predictable behavioural patterns present an opportunity to explore the neural responses associated with successful and unsuccessful/impaired discrimination.

Mismatch negativity (MMN) refers to an experimental paradigm which has been extensively applied to investigate sensory processing. In a MMN paradigm, standard stimuli are presented repeatedly in relatively close succession. When the standard sequence is punctuated with a discriminably different stimulus (e.g. a change or absence), known as a deviant stimulus, measurable neural activity is automatically generated. When a MMN paradigm is applied during electroencephalography (EEG) recording, the activity is seen as the MMN component - a negative deflection in the event related potential, peaking $150-200 \mathrm{~ms}$ after the onset of the stimulus (Kropotov et al., 1995; Näätänen, Kujala, \& Winkler, 2011). The magnitude (amplitude) of the MMN response, like other ERP components, reflects the size and/or synchronicity of the local neuronal populations generating the activity (Gruber, Klimesch, Sauseng, \& Doppelmayr, 2004; Tremblay, Kraus, McGee, Ponton, \& Otis, 2001). Robson et al. (2014) recorded EEG while presenting a multiple deviant MMN paradigm to participants with WA. MMN ERP amplitude was analysed following the presentation of behaviourally perceived 
and non-perceived phoneme changes. ERP amplitude was not found to differ depending on whether the phoneme change was behaviourally perceived or not, indicating that behavioural performance was not accounted for by activity in local neuronal populations responding to the acoustic structure of the stimuli. This lack of difference was also seen in matched neurotypical participants presented with between phonemic category (perceived) and within phonemic category (non-perceived) changes. This pattern replicated previous studies contrasting between and within-category phoneme changes in control participants. In these studies it was interpreted that the MMN ERP reflected acoustic but not phonetic and phonological analysis of the stimuli (Robson et al., 2014; Sharma, Kraus, McGee, Carrell, \& Nicol, 1993).

Therefore, it is not altogether unsurprising that a MMN ERP analysis was unable to distinguish intact and impaired phonological analysis in WA. While accurate acoustic analysis of the speech stream is necessary for phoneme perception, it is not in itself sufficient. Successful speech perception requires recovery of a discrete code from an acoustic signal that varies between speaker and linguistic contexts. As such, the speech perception system must abstract away from this surface variability in order to recover the intended message. The hypothesised process by which the speech perception network achieves this abstraction is known as hierarchical processing (Davis \& Johnsrude, 2003; Humphries, Sabri, Lewis, \& Liebenthal, 2014). Hierarchical processing describes the function of regions within a perception network, whereby early components of the network are sensitive to structural stimulus properties, e.g. the acoustic components of phonemes, and higher-order regions of the network are sensitive to invariant stimulus properties e.g. phonemic category. Higher order regions are proposed to achieve this abstraction through "combination sensitivity" in neuronal tuning; that is, sensitivity to patterns of responses across lower-order regions (DeWitt \& Rauschecker, 2012; Shamma, 2014). This combination sensitivity enables these network components to respond similarly to different exemplars of acoustic-phonetic information thus abstracting away from lower-order surface information (Carruthers et al., 2015; Mesgarani, Cheung, Johnson, \& Chang, 2014). This 
model has emerged from experimental observations, for example, that MMN (acoustic) responses to a range of stimuli are associated with activity in and surrounding the primary auditory cortices (Alho, 1995; Molholm, Martinez, Ritter, Javitt, \& Foxe, 2005; Opitz, Rinne, Mecklinger, von Cramon, \& Schröger, 2002), whereas identification of phonemic category independent of acoustic structure is associated with a range of higher order regions, including the posterior superior temporal gyrus and sulcus, inferior parietal and inferior frontal lobes (Chang et al., 2010; Joanisse, Zevin, \& McCandliss, 2007; Liebenthal et al., 2010; Liebenthal, Binder, Spitzer, Possing, \& Medler, 2005; Myers, Blumstein, Walsh, \& Eliassen, 2009). Based on this model, Robson et al. (2014) hypothesised that the behavioural impairment in WA may be better characterised by disruption in activity across the hierarchical speech perception network, or the ability to accurately analyse, integrate and transfer information.

The current study directly evaluates the hierarchical processing impairment hypothesis by examining oscillatory activity following phoneme changes, i.e. activity in the MMN window. Hierarchical processing requires information transfer between neural populations within and potentially between cognitive networks. Neural activity is generated by the repeated spiking of neurons - neural oscillations. This generates local field potentials which can be measured by electrophysiology techniques. If two populations of neurons spike at the same time and rate, they will produce the same frequency of oscillatory activity which is in phase. If neural populations spike at the same rate but at different times they will produce the same frequency of oscillatory activity but this activity will be out of phase. Activity from within a neural population is proposed to be in phase, whereas an interaction between neurons requires a time lag and, thus, oscillatory activity between these neuronal populations would be out of phase (Nolte et al., 2004; Weisz \& Obleser, 2014). Direct evidence for time-lagged inter-neuronal population interactions has been found using local field potential recordings in rats in which a consistent lag between oscillatory activity in the same frequency band during task performance (Siapas, Lubenov, \& Wilson, 2005). The identification of such inter-neuronal population 
interactions within the stroke population is methodologically challenging. At the sensor level, non-instantaneous activity is masked by volume conduction and the reconstruction of sourcelevel activity is confounded by the presence of lesion. To address these challenges this study applied the non-zero phase lagged (NZPL Drakesmith, El-Deredy, \& Welbourne, 2013) methodology to sensory level EEG data to observe oscillatory activity while removing the effects of volume conduction. In this method, oscillatory activity - measured in power (amplitude ${ }^{2}$ ) requires the decomposition of the EEG signal into constituent frequencies using a Fourier transform. This transform results in both amplitude and phase information of oscillatory activity at constituent frequencies. The phase of an oscillation at a given sensor can be compared to all other sensors. The NZPL analysis isolates the power of oscillatory activity that has some degree of measurable phase-lag (on the order of milliseconds) in comparison to oscillatory activity of the same frequency recorded at other sensors. This measure is thought to better identify the degree of inter-neuronal population communication, given that activity from within a neuronal population or that associated with the effects of volume conduction has no phase lag and is removed from the analysis (see Drakesmith et al., 2013 and supplementary materials for methodological details). As such, NZPL power is not a form of functional connectivity between source regions but rather a measure of the degree of activity measured at sensor level which is associated with communication between neuronal populations.

It was hypothesised that the presentation of perceptible phoneme changes would result in an increase in NZPL power under the assumption that phoneme changes are perceived when phonological information is extracted following hierarchical auditory analysis and that this analysis requires information transfer between neuronal populations. Secondly, it was hypothesised that non-perceptible phoneme/phonetic changes would be insufficiently different to generate new hierarchical auditory activity in comparison to established auditory activity and, therefore, these changes are hypothesised to generate little or no change to NZPL power and significantly reduced NZPL power in comparison to perceptible changes. These hypotheses 
are within subject and participants with WA and neurotypical participants were predicted to display the same patterns when exposed to stimuli which generated matched behavioural performance. Importantly these hypotheses differ from the MMN ERP patterns which showed no difference between responses to perceived and non-perceived phoneme changes. The NZPL power was hypothesised to be sensitive to behavioural phoneme change perception because, unlike the MMN ERP, it is sensitive to communication between neuronal populations and it is this integrated activity across the hierarchical network which is proposed to give rise to the behavioural percept.

\section{$\underline{\text { Methods }}$}

Ethical approval was provided by the University of Manchester ethics committee. Seven individuals (all male) with Wernicke's aphasia (WA) and seven age-matched neurotypical participants (one female) were recruited from a panel of volunteers, Table 1. Informed consent was obtained for all individual participants in the study. Data collection took place at the University of Manchester.

\section{Participants}

Eligibility criteria for the WA participants were a chronic (greater than 9 months) diagnosis of WA following a single cerebrovascular accident (CVA). Diagnosis was based on the Boston Diagnostic Aphasia Examination - Short Form (Goodglass, Kaplan, \& Barresi, 2001) and clinical opinion of the first author which took into account motor and speech error profile (no or limited motor disruption, significant proportion of phonological errors). Exclusion criteria were the presence of significant neurological history beyond the single CVA and history of drug or alcohol abuse. Eligibility criteria for neurotypical participants was age and education matching to WA participants and indication of typical ageing according to the ACE-R (Mioshi, Dawson, Mitchell, Arnold, \& Hodges, 2006). 3T T1-weighted MRI scans and corresponding lesion data were available for 6/7 WA participants and a lesion overlap map for these individuals is displayed in 
Figure 1. Total lesion overlap $(n=6)$ was found in the posterior superior temporal gyrus and posterior-mid superior temporal sulcus.

Figure 1: Lesion Overlap Map

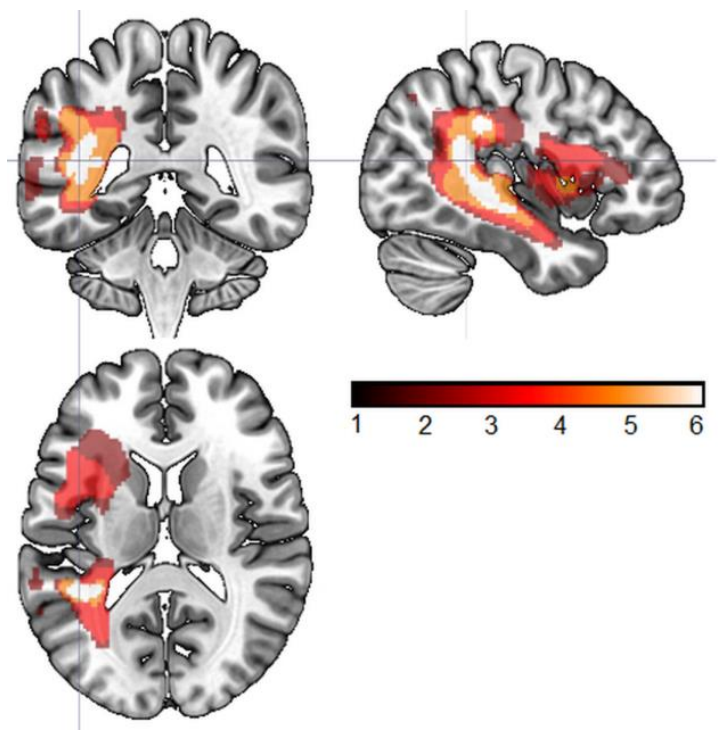

LOM for 6/7 WA participants at MNI coordinates $-42-41$ 14. Colour bar indicates number of participants with lesion at each voxel. Created in MRIcro GL and thresholded at $n=3$.

\section{Outcome Variable}

This study analysed the Non-Zero Phase Lagged (NZPL) power of the EEG signal at each sensor (Drakesmith et al., 2013) which occurred following behaviourally perceived and non-perceived phonemic/phonetic changes elicited through a multiple deviant mismatch negativity (MMN) paradigm. 
Table 1: Participant Demographics and Lesion Location

\begin{tabular}{|c|c|c|c|c|c|c|c|c|}
\hline & \multirow[b]{2}{*}{ PT } & \multirow[b]{2}{*}{ TPO } & \multirow[b]{2}{*}{ Age } & \multicolumn{3}{|c|}{ BDAE screening profile } & \multirow[b]{2}{*}{$\begin{array}{l}\text { ACE-R } \\
(\max .100)\end{array}$} & \multirow[b]{2}{*}{ Lesion location } \\
\hline & & & & $\begin{array}{l}\text { Flu. } \\
(\%)\end{array}$ & $\begin{array}{l}\text { Rep. } \\
(\%)\end{array}$ & $\begin{array}{l}\text { Comp. } \\
(\%)\end{array}$ & & \\
\hline \multirow{7}{*}{ 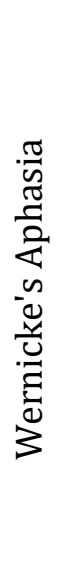 } & 1 & 20 & 77 & 47 & $<1$ & 2 & $\mathrm{n} / \mathrm{a}$ & pSTG; p,mSTS; pMTG; SMG; Ins; IFL \\
\hline & 2 & 25 & 68 & 47 & $<1$ & 3 & $\mathrm{n} / \mathrm{a}$ & $\begin{array}{l}\text { p,mSTG; p,mSTS; p,mMTG; AG; } \\
\text { SMG; Ins; IFL }\end{array}$ \\
\hline & 3 & 23 & 74 & 63 & $<1$ & 5 & $\mathrm{n} / \mathrm{a}$ & p,m,aSTG; p,m,aSTS; SMG; Ins; IFL \\
\hline & 4 & 34 & 69 & 75 & 10 & 14 & $\mathrm{n} / \mathrm{a}$ & p,mSTG; p,mSTS; mMTG; SMG; AG \\
\hline & 5 & 24 & 67 & 70 & 22.5 & 18 & $\mathrm{n} / \mathrm{a}$ & $\begin{array}{l}\text { p,m,aSTG; p,m,aSTS; m,aMTG; SMG; } \\
\text { Ins; IFL }\end{array}$ \\
\hline & 6 & 33 & 67 & 68 & 22.5 & 20 & $\mathrm{n} / \mathrm{a}$ & $\mathrm{N} / \mathrm{A}$ \\
\hline & 7 & 54 & 72 & 100 & 22.5 & 42 & $\mathrm{n} / \mathrm{a}$ & mSTG; p,m,aSTG; SMG \\
\hline \multirow{7}{*}{ 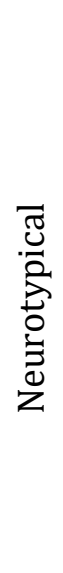 } & 1 & $\mathrm{n} / \mathrm{a}$ & 73 & $\mathrm{n} / \mathrm{a}$ & $\mathrm{n} / \mathrm{a}$ & $\mathrm{n} / \mathrm{a}$ & 92 & \\
\hline & 2 & $\mathrm{n} / \mathrm{a}$ & 70 & $\mathrm{n} / \mathrm{a}$ & $\mathrm{n} / \mathrm{a}$ & $\mathrm{n} / \mathrm{a}$ & 98 & \\
\hline & 3 & $\mathrm{n} / \mathrm{a}$ & 73 & $\mathrm{n} / \mathrm{a}$ & $\mathrm{n} / \mathrm{a}$ & $\mathrm{n} / \mathrm{a}$ & 95 & \\
\hline & 4 & $\mathrm{n} / \mathrm{a}$ & 69 & $\mathrm{n} / \mathrm{a}$ & $\mathrm{n} / \mathrm{a}$ & $\mathrm{n} / \mathrm{a}$ & 99 & \\
\hline & 5 & $\mathrm{n} / \mathrm{a}$ & 77 & $\mathrm{n} / \mathrm{a}$ & $\mathrm{n} / \mathrm{a}$ & $\mathrm{n} / \mathrm{a}$ & 78 & \\
\hline & 6 & $\mathrm{n} / \mathrm{a}$ & 61 & $\mathrm{n} / \mathrm{a}$ & $\mathrm{n} / \mathrm{a}$ & $\mathrm{n} / \mathrm{a}$ & 92 & \\
\hline & 7 & $\mathrm{n} / \mathrm{a}$ & 60 & $\mathrm{n} / \mathrm{a}$ & $\mathrm{n} / \mathrm{a}$ & $\mathrm{n} / \mathrm{a}$ & 97 & \\
\hline
\end{tabular}

PT num. = participant number; TPO = time post onset in months; BDAE = Boston Diagnostic Aphasia Examination (Goodglass et al., 2001); Flu. (\%) = Fluency centile rank; Rep. (\%) = Repetition centile rank; Comp. $(\%)=$ Comprehension centile rank; ACE-R = Addenbrooks Cognitive Examination Revised (Mioshi et al., 2006); Lesion location in language related areas: $\mathrm{p}=$ posterior; $\mathrm{m}=$ mid; $\mathrm{a}=$ anterior; $\mathrm{STG}$ = superior temporal gyrus; $\mathrm{STS}$ = superior temporal sulcus; MTG = middle temporal gyrus; $\mathrm{SMG}$ = supramarginal gyrus; $\mathrm{AG}$ = angular gyrus; $\mathrm{Ins}=$ insula; $\mathrm{IFL}=$ inferior frontal lobe

\section{Identification of Perceived and Non-Perceived Phonetic Contrasts}

The phonetic changes presented to the WA and neurotypical participants differed to reflect the behavioural impairment present in WA. A modified version of the adaptive phonological 
discrimination test (Robson, Keidel, et al., 2012) which included within phonemic category contrasts was used to establish consistently perceived and consistently non-perceived phonemic/phonetic contrasts in each group. The adaptive phonological discrimination test consisted of 9 difficulty levels of CVC non-word discrimination. On each trial the participant was presented with three non-words in $\mathrm{AxB}$ format and were asked to identify (non-verbally, by pointing) which stimulus was the odd-one-out. They were informed that the odd-one-out was never in the central position. Levels $9-5$ presented between phonemic category contrasts from low to high confusability based on Miller and Nicely's (1955) confusability ratings. Levels 4-1 used within phonemic category phonetic changes with decreasing acoustic difference between the reference and target stimuli. Within category differences were produced in Praat using the PSOLA algorithm to create 10 step continua between audio recorded exemplars of /p/ \& /b/ and $/ \mathrm{p} /$ \& $/ \mathrm{k} /$. Level 4 of the phonological discrimination assessment presented CVC nonwords in which the initial phoneme had the largest within-category acoustic difference (between steps $10 \& 6$ and $1 \& 5$ of the continua). Level 1 of the assessment presented stimuli with the smallest within-category acoustic difference (between steps $10 \& 9$ and $1 \& 2$ of the continua). The test used a three-down one-up adaptive tracking design and was discontinued after 8 level reversals. The threshold level at which phoneme changes could be identified was calculated as the average of the level at which the final 4 reversals occurred. Only at the lowest confusability levels (level 9) were phoneme changes consistently perceived for all WA participants. Therefore perceptible changes consisted of phonological contrasts with large acoustic differences e.g. /k/-/v/. Neurotypical participants could consistently perceive the smallest between category acoustic changes e.g. /p/-/k/ (level 5). These perceptible changes in the neurotypical group were consistently not perceived by the WA group and, therefore, were used as the WA group's non-perceptible contrast. In the neurotypical group, non-perceptible contrasts were formed of within-phonemic category changes matched for acoustic difference to the between category contrasts. 
EEG data collection and preprocessing

EEG was recorded in an electrically shielded (but not sound proof) room while two passive ERP paradigms were presented: a multiple deviant oddball paradigm and a "deviant alone" paradigm. The paradigms were presented using Eprime 1.0 (Psychology Tools, www.pstnet.com). The oddball paradigm presented 743 pseudo-randomised, jittered non-word consonant-vowel-consonant (CVC) stimuli. Three triplets of standard, perceptible and nonperceptible stimulus were presented to each group in separate runs (see supplementary materials for stimuli). As above, perceptible stimuli were non-word CVC stimuli which could be detected as different to the standard non-word CVC stimuli. Non-perceptible stimuli were nonword CVC stimuli which could not be detected as different to the standard. Standard stimuli were presented on $65 \%$ of occurrences, deviants which formed either perceptible or nonperceptible changes from the standard stimuli were presented on $17.5 \%$ of occurrences ( $n=130 /$ deviant condition). The deviants were additionally presented as standards in a "deviant alone" condition, which consisted of six runs of repeated presentation of only one deviant stimulus. A deviant-alone condition allows the examination of the effects of phoneme change in comparison to the same stimulus presented in a no-change condition. Auditory stimuli were presented through headphones at 78dB SPL. Participants watched a silent nature programme while the paradigms were presented.

EEG data were collected with a 64 electrode (10-20 configuration) Biosemi ActiveTwo system and Actiview acquisition software (Biosemi, Netherlands) referenced using the online biosemi feedback loop system (cf. http://www. biosemi.com/faq/cms\&drl.htm) at a sampling rate of $512 \mathrm{~Hz}$. EEG channels were recorded if impedance was below $20 \mathrm{k} \Omega$. If the impedance could not be brought below this level, the channel was turned off and data were interpolated from surrounding channels prior to pre-processing. The ERP data were pre-processed using the EEGlab Matlab toolbox (Delorme and Makeig 2004). EEG data were divided into epochs from - 
$100-600 \mathrm{~ms}$ and baseline corrected (baseline period -100 to $0 \mathrm{~ms}$ ). Time $0 \mathrm{~ms}$ corresponded to the onset of the CVC stimuli. Data were re-referenced to the average of all electrodes and bandpass filtered $2-40 \mathrm{~Hz}$ offline. Independent component analysis was performed and components related to eye blinks subtracted from the data. Finally, epochs containing amplitudes of greater than $120 \mu \mathrm{V}$ were removed.

\section{NZPL calculation}

Non-zero phase lag (NZPL) power was then calculated using the preprocessed data by taking the imaginary components of the cross spectral density matrix and then performing an eigenvalue decomposition to isolate the NPZL components of the power (see supplementary material for further details). The NZPL power was averaged in four frequency bands $(4-8 \mathrm{~Hz}, 8-$ $12 \mathrm{~Hz}, 12-24 \mathrm{~Hz}$ and $24-40 \mathrm{~Hz}$ ) across all remaining deviant trials in each condition (oddball perceived change; oddball non-perceived change; deviant alone perceived; deviant alone nonperceived). The average ERP was divided into three time segments - central MMN, pre MMN and post MMN - and the NZPL signal was averaged within these time segments. The pre MMN segment was $75-125 \mathrm{~ms}$ after stimulus onset, while the post MMN segment was $375-425 \mathrm{~ms}$ after stimulus onset. The central MMN segment spanned 150-250ms post stimulus onset. Values 2 standard deviations above the mean were removed and interpolated from surrounding sensors. Preparation for statistical analysis involved subtracting the NZPL power in standard conditions (deviant alone) from the deviant (oddball) signal to identify the power difference between when a syllable was presented in a change condition to when the same syllable was presented in a non-change condition. In order to compare across stimuli and power bands the remaining epochs were normalised. The relative power in each segment was calculated by dividing the segment power by the mean absolute NZPL power of the three segments. Finally, MMN NZPL power change (henceforth MMN signal) was calculated by subtracting the mean relative signal in the pre and post MMN segments from the central MMN segment. Normality tests were conducted on the MMN signal averaged over all sensors. Normally distributed data 
were observed for each group, frequency band and perceptibility condition. Therefore, the MMN signal represents the integrated neural activity associated with perceived and nonperceived phoneme change relative to the activity within the same trial and that which occurs over the same time period following the presentation of the same stimulus in a deviant alone/no-change condition.

Figure 2: Figure 2: Sensor Layout for NZPL power analysis

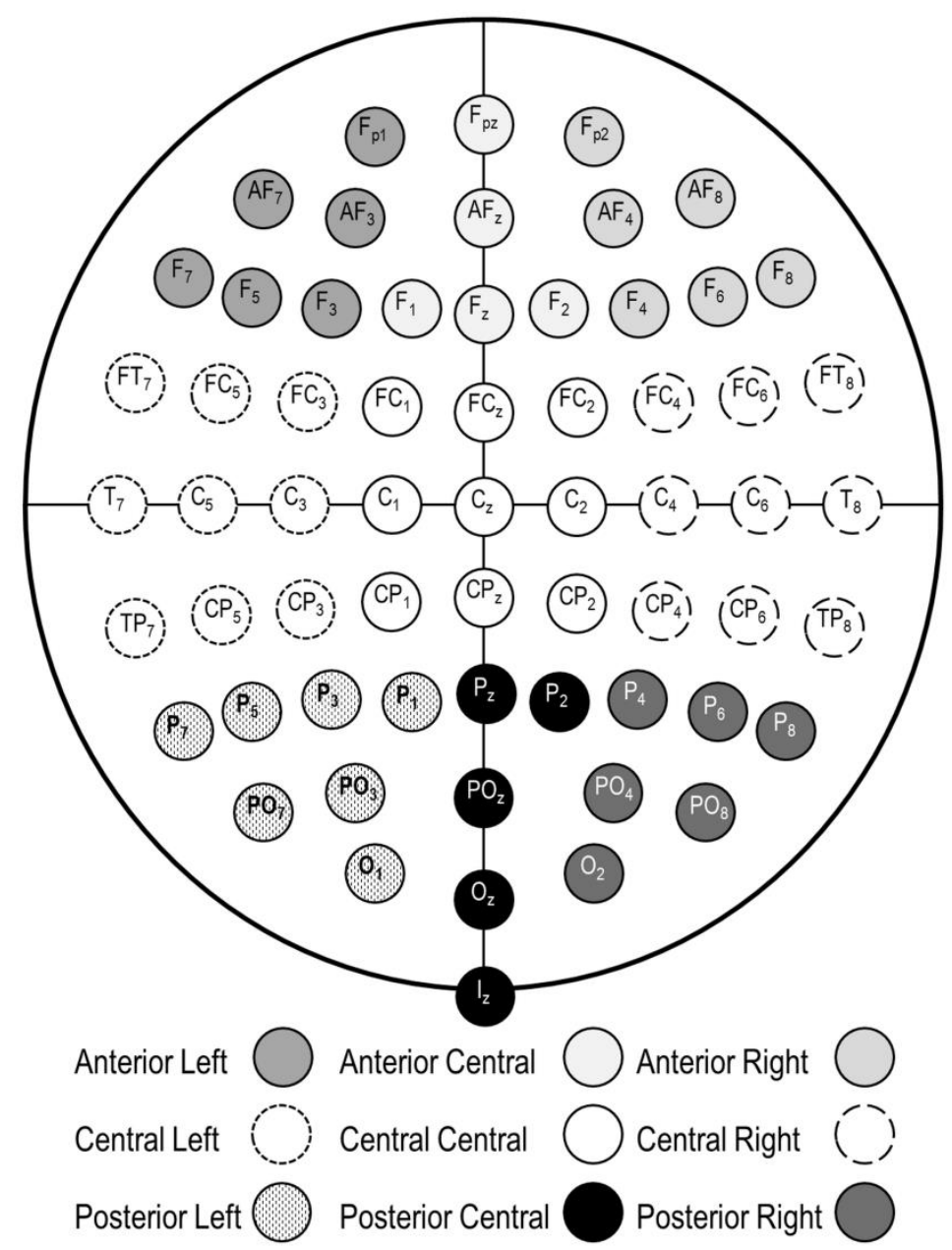

Figure indicates grouping of sensors in NZPL power analysis. NB sensors P9 and P10 not shown within posterior left and posterior right groupings, respectively. Created in MS Powerpoint. 


\section{Statistical Analyses}

The MMN signal from all sensors for perceptible phoneme change stimuli in control participants was averaged and submitted to four one-tailed, one-sample t-tests corresponding to each of the frequency bands. This revealed that the MMN signal was significantly above zero for the 4-8, 812 and $12-24 \mathrm{hz}$ bands $\left(\mathrm{t}_{(6)}=1.98, \mathrm{p}=0.05 ; \mathrm{t}_{(6)}=2.7, \mathrm{p}=0.02 ; \mathrm{t}_{(6)}=1.9, \mathrm{p}=0.05\right.$, respectively) but not for the $24-40 \mathrm{hz}$ band $\left(\mathrm{t}_{(6)}=1.1, \mathrm{p}=0.15\right)$. In subsequent analyses only frequency bands were included for which the MMN signal was detectable in the neurotypical participants. The MNN signal was then averaged into nine groups of sensors split into a three-by-three grid on the anterior to posterior, and left to right axes (Figure 2). To test the primary objective and hypothesis that inter-neuronal population communication increases with perceptible but not non-perceptible changes in both the WA and neurotypical participants, the average MMN signal from central electrodes (Area CC, Figure 2) was entered as the dependent variable into an ANOVA with phoneme change perceptibility (perceptible vs. non-perceptible) as a withinsubject factor and group as a between-subject factor.

\section{$\underline{\text { Results }}$}

In line with the hypothesis, the $2 x 2$ ANOVA (group x perceptibility) revealed a main effect of perceptibility $\left(\mathrm{F}_{1,12}=6.8\right.$, partial eta ${ }^{2}=.36, \mathrm{p}=0.023$; $\mathrm{MMN}$ Signal perceptible stimuli mean $=0.7$ $\mathrm{SD}=0.8 ; \mathrm{MMN}$ Signal non-perceptible stimuli mean $=-0.6 \mathrm{SD}=2.2$ ). A borderline group $\mathrm{x}$ perceptibility interaction was also observed $\left(\mathrm{F}_{1,12}=3.3\right.$, partial eta $\left.{ }^{2}=.22, \mathrm{p}=0.095\right)$. To explore this interaction and the wider spatial distribution of the MMN Signal, the data further the MMN signal split by the anterior-posterior and left-right axes together with perceptibility and group is displayed in Figure 3. In the neurotypical participants, a widespread positive MMN signal was observed in anterior, central and posterior areas in both hemispheres in the perceptible change condition, i.e. these regions produced increased NZPL power to perceptible phoneme changes. This positive MMN Signal was significant in the central region. In the WA participants, positive MMN signal change was also detect in response to perceptible phoneme changes and this was 
significant at the group level in the left anterior and central region. In comparison to the neurotypical group, the WA participants displayed more limited MMN Signal in the right hemisphere and posterior regions. Non-perceptible phoneme changes in the WA group produced a negative MMN signal in almost all sensor regions and this was significant in anterior and central right hemisphere regions. A negative MMN signal indicates a decrease in interneuronal population activity to following phoneme change in comparison to the signal generated when the same phoneme was repeated as a standard stimulus in the deviant alone/no-change condition.

Figure 3: NZPL power change results

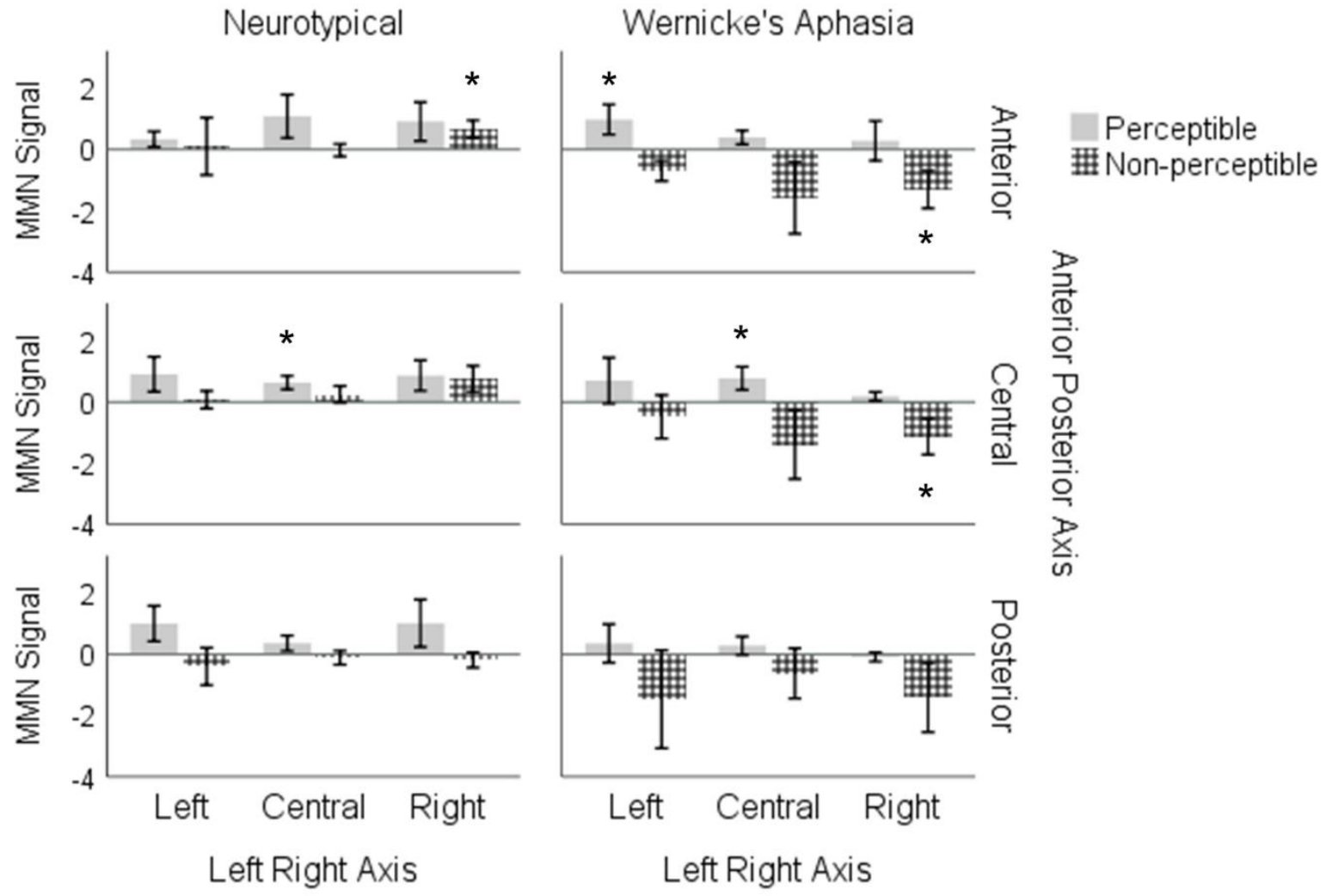

MMN Signal = NZPL power mismatch negativity signal. Mean MMN Signal shown for Wernicke's aphasia and neurotypical participants following changes to a repeated CVC stimulus that was either a perceptible or non-perceptible change in comparison to the standard stimulus. ${ }^{*}$ indicate significantly different from zero at alpha $=0.05$, one-tailed. No region showed significant MMNS after Bonferroni correction for multiple comparisons. Created in SPSS. 


\section{$\underline{\text { Discussion }}$}

This study examined the theory that the behavioural percept of phoneme change emerges secondary to integrated activity throughout the hierarchical speech perception network. It was hypothesised that phoneme changes that were perceptible to individuals with Wernicke's aphasia (WA), presented during a mismatch negativity (MMN) oddball paradigm, would generate increased integrated neural activity in comparison to when the same stimuli were presented in a deviant alone/no-change condition. In contrast, phoneme changes that were not perceptible, reflecting impaired phonological processing, would be associated no or limited increases in integrated activity. These patterns were also expected in the neurotypical population in response to between category (perceived) and within category (non-perceived) phoneme/phonetic changes. To explore these hypotheses this study used an EEG non-zero phase lagged (NZPL) power analysis to isolate the EEG power associated with inter-neuronal population activity rather than local neuronal population activity. Conforming to the hypotheses, this study found that a significant difference in the NZPL signal depending on whether phoneme changes were perceptible or non-perceptible. The ability to perceive a phoneme change is associated with increased NZPL power in both the typical speech perception of the neurotypical participants and the pathologically impaired speech perception of WA. Nonperceived phonetic/phonemic changes resulted in more limited NZPL power change in neurotypical participants and an unexpected reduction in NZPL power in WA participants. These results are some of the first to contribute to the mechanistic underpinnings differentiating impaired and intact behaviour within an individual with chronic WA, a condition associated with auditory comprehension impairments secondary to speech perception disruption.

Previous ERP analysis of the data from the current study was not able to discriminate behavioural differences between the perceived and non-perceived phoneme change condition, 
both of which elicited similar ERP activity (Robson et al., 2014). These results were consistent with the interpretation of the MMN as a marker of pre-conscious acoustic change perception (Näätänen et al., 2011) and confirming that early acoustic responses are not equivalent to, or sufficient for, conscious perception of phoneme change. Subsequently it was hypothesised that the success of behavioural phoneme change detection would be reflected in NZPL power, an EEG measure which removes the effects of volume conduction (Drakesmith et al., 2013) to reveal non-instantaneous neuronal activity, thought to reflect interaction between populations of neurons (Nolte et al., 2004). The current study used NZPL power as the outcome measure in a standard MMN analysis. That is, the NZPL power generated in response to repeated "deviant alone" stimuli/no-change phonemes was subtracted from the NZPL power generated following the presentation of the same stimuli presented as deviants in the oddball paradigm/change phonemes. Thus, the resulting values reflect the change in the degree of integrated activity following perceptible and non-perceptible phoneme change. In the neurotypical participants, perceptible between-category phoneme change was associated with an enhancement in integrated activity where as non-perceptible within-category phoneme change resulted in more limited change to integrated activity in comparison to the standard stimulus. These results are interpretable within a hierarchical auditory processing model. In accordance with this model, non-perceptible within category changes presented to the neurotypical group generated MMN ERP activity reflecting a change in the acoustic structure. However, the acoustic difference between these stimuli remained within the same combinatorial sensitivity window, resulting in more limited changes in activity power across the wider network and more limited change in NZPL power. In contrast, acoustic-phonetic changes that fell outside the established combinatorial sensitivity window (perceptible between-category changes) elicited both a MMN and significantly enhanced NZPL power, indicating enhanced transfer of information through the network. These results support hierarchical processing theories as a plausible mechanism which allows the extraction of linguistically relevant information while simultaneously preventing non-relevant acoustic variation from dominating conscious perception. Although 
NZPL power change to non-perceived within-category changes was more limited, significantly increased activity was observed in right anterior sensor regions in the neurotypical group. Increased activity was also observed in this region to perceived phoneme changes, although greater variation in the group meant that this did not reach significance. Right anterior sensor activity is commonly observed in EEG MMN paradigms and associated with involuntary attention switching (Rinne, Alho, Ilmoniemi, Virtanen, \& Näätänen, 2000). This may account for this finding, given that both the perceived and non-perceived changes generate acoustic activity. Individuals with WA also displayed increased in NZPL power following phoneme changes. Although individuals with WA present with significant impairments in phonological analysis, some capacity to perform phonological discrimination tasks when acoustic differences between phonemes/words are sufficiently large (Robson, Sage, et al., 2012). Taken together with the previous ERP study, these data suggest that retained ability may be underpinned by the capacity to transfer information through the hierarchical speech perception network, rather than magnitude of acoustic response to phonological information.

Perceptible phoneme change elicited significantly increased power, in comparison to the standard no-change condition, in central sensors in both groups - the scalp region associated with maximum signal in auditory electrophysiology studies (Näätänen et al., 2011). The WA group also displayed significant activity in the left anterior sensor set. The MMN Signal did not reach significance in other sensor sets, although the neurotypical group displayed some degree of activity in the majority of areas and, therefore, this may reflect statistical power rather than lack of effects. It is important to note that sensor-level activity shows low correspondence to the source location (Haufe, Nikulin, Müller, \& Nolte, 2013; Papadopoulou, Friston, \& Marinazzo, 2015; Van de Steen et al., 2016) and that source information cannot be inferred from the current study. However, it is interesting that limited MMN Signal was observed in right hemisphere sensor sets to perceptible changes in the WA participants. Given that individuals with WA have lesions consistently affecting the left superior temporal lobe (Figure 1), some 
observable activity in right hemisphere areas which remain intact might be expected. However, these results do not indicate an absence of activity, rather, that the activity measured at these right hemisphere sensors was similar in the phoneme change and no-change condition. Unexpectedly, these right hemisphere regions displayed a significant negative NZPL MMN Signal to non-perceptible changes (Figure 3).

The negative change in NZPL power to non-perceptible changes suggests that the WA produce less integrated activity when presented with a phoneme change that they do not readily perceive in comparison to when the same phoneme is presented repeatedly in a no-change condition. Findings from the previous MMN ERP study revealed that although non-perceptible phonemic changes elicited an MMN in the WA group, this MMN was significantly delayed in comparison to that generated by the same stimulus change in the neurotypical group (Robson et al., 2014). Accurate phonetic processing requires analysis of rapid acoustic transitions and integration of acoustic features presented over short time scales. This is particularly the case for stop consonants, which were used as the non-perceptible contrast in the WA group. It is hypothesised that disrupted temporal analysis of the acoustic signal may restrict the formation of an integrated feed-forward network response, particularly on the first stimulus presentation. In comparison, repeatedly presenting the same stimulus in the deviant alone condition may generate an increasingly integrated response throughout the auditory network and the development of a more stable integrated activation pattern. The contrast between repeated stimulus presentation and one-off stimulus change could, therefore, result in a relative reduction in neural integration in the change condition. Therefore, a percept of reduced stability may be formed on the initial presentation resulting in behavioural discrimination impairments. This reduction in integration was significant in right central and anterior sensor sets and. One interpretation is that the presence of lesion in the left superior temporal lobe (Figure 1) greater involvement of auditory and language regions which are non-specialised for phonological processing, particularly the analysis of phonetic information which requires high temporal 
resolution (Belin et al., 1998), such as the plosive consonants in the non-perceptible condition in the WA group. As above, it is important to note that these results do not speak to the amount of activation at these sensors but rather the degree of change in activity. As such, it is not that perceptible and non-perceptible phoneme changes generate activity in different sensors but that the success of perception is associated with changes to integrated neural activity recorded at the sensors. The results of the current study are consistent with a mMMN study undertaken in participants with left hemisphere superior temporal lobe lesions (Teki et al., 2013). Participants in this study also displayed altered network dynamics during phoneme perception, particularly in right hemisphere auditory structures. Taken together, these studies indicate that disordered network dynamics in response to changing phonemic information is a consistent hallmark of individuals with impaired speech perception and a potential biomarker of WA. Crucially, the observed pattern of enhanced integration in response to perceptible phoneme change in both the neurotypical and the WA group appeared to reflect behavioural capacity, rather than the phonemic stimuli themselves. The between-category changes perceptible to the neurotypical group were consistently not perceived in the WA group. Therefore, the betweencategory changes used as perceptible stimuli in the neurotypical group were used as nonperceptible stimuli in the WA group. Therefore, this presents the opportunity to investigate the impact of speech perception rehabilitation programmes at a neural level, allowing the exploration of whether therapies are "re-normalising" network dynamics or having an impact at other levels e.g. acoustic processing only.

This study aimed to investigate within-subject variation in speech perception in WA. Impaired speech perception is the level of cognitive disruption traditionally associated with the WA comprehension impairment (Eggert, 1977; Luria, 1976; Luria \& Hutton, 1977) and neuropsychological investigations have documented a systematic relationship between auditory and phonological processing and language comprehension in WA (e.g. Robson et al., 2017). Recent evidence indicates that this speech perception-auditory comprehension 
relationship emerges during the course of recovery and that the language comprehension network is more disorganised at the sub-acute phase than at the chronic phase (Robson, Griffiths, Grube, \& Woollams, 2019). This indicates that, while these results may explain behavioural symptoms at the chronic phase, they may not be generalizable to the sub-acute phase and before. In addition, it is important to note that the comprehension impairment in WA cannot be fully accounted for by disrupted speech perception processes and that impairments in sematic and executive processing are consistently documented in a large proportion of the WA population, as is consistent with the extended lesion profile (Ogar et al., 2011; Robson, Sage, et al., 2012; Thompson, Robson, Lambon Ralph, \& Jefferies, 2015). The results from the current study should be considered within this wider context and further efforts made to unpick and support the cognitive disruption contributing to this severely disabling language impairment.

\section{Conclusion}

In the neurotypical brain, the behavioural percept of phonemic changes is related to enhanced integrated neural responses. In comparison, acoustic-phonetic changes which are nonperceptible, e.g. those that fall within a phonemic category boundary, do not generate enhanced integrated activity despite generating early auditory responses. Individuals with pathologically impaired speech perception adhere to this pattern for behaviourally perceived phonemic contrasts. However, more acoustically similar, non-perceptible phonemic changes elicit reduced neural network integration. Impairments in producing integrated neural activity to new phonological information may be a biomarker of a disordered speech perception system.

\section{Ethical statement}

All procedures performed in studies involving human participants were in accordance with the ethical standards of the institutional and/or national research committee and with the 1964 Helsinki declaration and its later amendments or comparable ethical standards 


\section{$\underline{\text { References }}$}

Alho, K. (1995). Cerebral generators of mismatch negativity (MMN) and its magnetic counterpart (MMNm) elicited by sound changes. Ear and Hearing, 16(1), 38-51.

Belin, P., Zilbovicius, M., Crozier, S., Thivard, L., Fontaine, and A., Masure, M.-C., \& Samson, Y. (1998). Lateralization of Speech and Auditory Temporal Processing. Journal of Cognitive Neuroscience, 10(4), 536-540. http://doi.org/10.1162/089892998562834

Carruthers, I. M., Laplagne, D. A., Jaegle, A., Briguglio, J. J., Mwilambwe-Tshilobo, L., Natan, R. G., \& Geffen, M. N. (2015). Emergence of invariant representation of vocalizations in the auditory cortex. Journal of Neurophysiology, 114(5), 2726 LP-2740. http://doi.org/10.1152/jn.00095.2015

Chang, E. F., Rieger, J. W., Johnson, K., Berger, M. S., Barbaro, N. M., \& Knight, R. T. (2010). Categorical speech representation in human superior temporal gyrus. Nat Neurosci, 13(11), 1428-1432. Retrieved from http://dx.doi.org/10.1038/nn.2641

Davis, M. H., \& Johnsrude, I. S. (2003). Hierarchical processing in spoken language comprehension. Journal of Neuroscience, 23(8), 3423-3431. http://doi.org/10.1523/JNEUROSCI.23-08-03423.2003

DeWitt, I., \& Rauschecker, J. P. (2012). Phoneme and word recognition in the auditory ventral stream. Proceedings of the National Academy of Sciences, 109(8), E505-E514. http://doi.org/10.1073/pnas.1113427109

Drakesmith, M., El-Deredy, W., \& Welbourne, S. (2013). Reconstructing coherent networks from electroencephalography and magnetoencephalography with reduced contamination from volume conduction or magnetic field spread. PLOS ONE, 8(12). http://doi.org/10.1371/journal.pone.0081553

Eggert, G. H. (1977). Wernicke's works on aphasia: a sourcebook and review. Mouton de Gruyter. Goodglass, H., Kaplan, E., \& Barresi, B. (2001). Boston Diagnostic Aphasia Examination, 3rd Edition, (BDAE). Baltimore: Lippincott Williams \& Wilkins.

Gruber, W. R., Klimesch, W., Sauseng, P., \& Doppelmayr, M. (2004). Alpha Phase Synchronization 
Predicts P1 and N1 Latency and Amplitude Size. Cerebral Cortex, 15(4), 371-377.

http://doi.org/10.1093/cercor/bhh139

Haufe, S., Nikulin, V. V, Müller, K.-R., \& Nolte, G. (2013). A critical assessment of connectivity measures for EEG data: a simulation study. Neuroimage, 64, 120-133.

Humphries, C., Sabri, M., Lewis, K., \& Liebenthal, E. (2014). Hierarchical organization of speech perception in human auditory cortex. Frontiers in Neuroscience, 8(December), 1-12. http://doi.org/10.3389/fnins.2014.00406

Joanisse, M. F., Zevin, J. D., \& McCandliss, B. D. (2007). Brain mechanisms implicated in the preattentive categorization of speech sounds revealed using fMRI and a short-interval habituation trial paradigm. Cerebral Cortex, 17(9), 2084-2093. http://doi.org/10.1093/cercor/bhl124

Kropotov, J. D., Näätänen, R., Sevostianov, A. V, Alho, K., Reinikainen, K., \& Kropotova, O. V. (1995). Mismatch negativity to auditory stimulus change recorded directly from the human temporal cortex. Psychophysiology, 32(4), 418-422. http://doi.org/10.1111/j.14698986.1995.tb01226.x

Liebenthal, E., Binder, J. R., Spitzer, S. M., Possing, E. T., \& Medler, D. A. (2005). Neural Substrates of Phonemic Perception. Cerebral Cortex, 15(10), 1621-1631. Retrieved from http://dx.doi.org/10.1093/cercor/bhi040

Liebenthal, E., Desai, R., Ellingson, M. M., Ramachandran, B., Desai, A., \& Binder, J. R. (2010). Specialization along the left superior temporal sulcus for auditory categorization. Cerebral Cortex, 20(12), 2958-2970. http://doi.org/10.1093/cercor/bhq045

Luria, A. R. (1976). Disturbances of understanding of verbal communication in patients with sensory aphasia. The Hague: Mouton \& Co. BV.

Luria, A. R., \& Hutton, J. T. (1977). A modern assessment of the basic forms of aphasia. Brain and Language, 4(2), 129-151. http://doi.org/http://dx.doi.org/10.1016/0093934X(77)90012-8

Mesgarani, N., Cheung, C., Johnson, K., \& Chang, E. F. (2014). Phonetic Feature Encoding in 
Human Superior Temporal Gyrus. Science, 343(6174), 1006 LP-1010. Retrieved from http://science.sciencemag.org/content/343/6174/1006.abstract

Miller, G. A., \& Nicely, P. E. (1955). An analysis of perceptual confusions among some English consonants. The Journal of the Acoustical Society of America, 27(2), 338-352. http://doi.org/10.1121/1.1907526

Mioshi, E., Dawson, K., Mitchell, J., Arnold, R., \& Hodges, J. R. (2006). The Addenbrooke's Cognitive Examination Revised (ACE-R): a brief cognitive test battery for dementia screening. International Journal of Geriatric Psychiatry, 21(11), 1078-1085. http://doi.org/10.1002/gps.1610

Molholm, S., Martinez, A., Ritter, W., Javitt, D. C., \& Foxe, J. J. (2005). The Neural Circuitry of Preattentive Auditory Change-detection: An fMRI Study of Pitch and Duration Mismatch Negativity generators. Cerebral Cortex, 15(5), 545-551. Retrieved from http://dx.doi.org/10.1093/cercor/bhh155

Myers, E. B., Blumstein, S. E., Walsh, E., \& Eliassen, J. (2009). Inferior Frontal Regions Underlie the Perception of Phonetic Category Invariance. Psychological Science, 20(7), 895-903. http://doi.org/10.1111/j.1467-9280.2009.02380.x

Näätänen, R., Kujala, T., \& Winkler, I. (2011). Auditory processing that leads to conscious perception: A unique window to central auditory processing opened by the mismatch negativity and related responses. Psychophysiology, 48(1), 4-22. http://doi.org/10.1111/j.1469-8986.2010.01114.x

Nolte, G., Bai, O., Wheaton, L., Mari, Z., Vorbach, S., \& Hallett, M. (2004). Identifying true brain interaction from EEG data using the imaginary part of coherency. Clinical Neurophysiology, 115(10), 2292-2307. http://doi.org/http://dx.doi.org/10.1016/j.clinph.2004.04.029

Ogar, J. M., Baldo, J. V, Wilson, S. M., Brambati, S. M., Miller, B. L., Dronkers, N. F., \& GornoTempini, M. L. (2011). Semantic dementia and persisting Wernicke's aphasia: Linguistic and anatomical profiles. Brain and Language, 117(1), 28-33. http://doi.org/http://dx.doi.org/10.1016/j.bandl.2010.11.004 
Opitz, B., Rinne, T., Mecklinger, A., von Cramon, D. Y., \& Schröger, E. (2002). Differential Contribution of Frontal and Temporal Cortices to Auditory Change Detection: fMRI and ERP Results. NeuroImage, 15(1), 167-174.

http://doi.org/http://dx.doi.org/10.1006/nimg.2001.0970

Papadopoulou, M., Friston, K., \& Marinazzo, D. (2015). Estimating directed connectivity from cortical recordings and reconstructed sources. Brain Topography, 1-12.

Rinne, T., Alho, K., Ilmoniemi, R. J., Virtanen, J., \& Näätänen, R. (2000). Separate Time Behaviors of the Temporal and Frontal Mismatch Negativity Sources. NeuroImage, 12(1), 14-19. http://doi.org/https://doi.org/10.1006/nimg.2000.0591

Robson, H., Cloutman, L., Keidel, J. L., Sage, K., Drakesmith, M., \& Welbourne, S. (2014). Mismatch negativity (MMN) reveals inefficient auditory ventral stream function in chronic auditory comprehension impairments. Cortex, 59, 113-125. http://doi.org/http://dx.doi.org/10.1016/j.cortex.2014.07.009

Robson, H., Griffiths, T. D., Grube, M., \& Woollams, A. M. (2019). Auditory, Phonological, and Semantic Factors in the Recovery From Wernicke's Aphasia Poststroke: Predictive Value and Implications for Rehabilitation. Neurorehabilitation and Neural Repair, 33(10), 800812. http://doi.org/10.1177/1545968319868709

Robson, H., Keidel, J. L., Lambon Ralph, M. A., \& Sage, K. (2012). Revealing and quantifying the impaired phonological analysis underpinning impaired comprehension in Wernicke's aphasia. Neuropsychologia, 50(2), 276-288.

http://doi.org/http://dx.doi.org/10.1016/j.neuropsychologia.2011.11.022

Robson, H., Pilkington, E., Evans, L., DeLuca, V., \& Keidel, J. L. (2017). Phonological and semantic processing during comprehension in Wernicke's aphasia: An N400 and Phonological Mapping Negativity Study. Neuropsychologia, 100, 144-154. http://doi.org/http://dx.doi.org/10.1016/j.neuropsychologia.2017.04.012

Robson, H., Sage, K., \& Lambon Ralph, M. A. (2012). Wernicke's aphasia reflects a combination of acoustic-phonological and semantic control deficits: A case-series comparison of 
Wernicke's aphasia, semantic dementia and semantic aphasia. Neuropsychologia, 50(2), 266-275. http://doi.org/http://dx.doi.org/10.1016/j.neuropsychologia.2011.11.021

Shamma, S. (2014). How phonetically selective is the human auditory cortex? Trends in Cognitive Sciences, 18(8), 391-392.

http://doi.org/http://dx.doi.org/10.1016/j.tics.2014.04.001

Sharma, A., Kraus, N., McGee, T., Carrell, T., \& Nicol, T. (1993). Acoustic versus phonetic representation of speech as reflected by the mismatch negativity event-related potential. Electroencephalography and Clinical Neurophysiology/Evoked Potentials Section, 88(1), 6471. http://doi.org/http://dx.doi.org/10.1016/0168-5597(93)90029-0

Siapas, A. G., Lubenov, E. V, \& Wilson, M. A. (2005). Prefrontal Phase Locking to Hippocampal Theta Oscillations. Neuron, 46(1), 141-151. http://doi.org/https://doi.org/10.1016/j.neuron.2005.02.028

Teki, S., Barnes, G. R., Penny, W. D., Iverson, P., Woodhead, Z. V. J., Griffiths, T. D., \& Leff, A. P. (2013). The right hemisphere supports but does not replace left hemisphere auditory function in patients with persisting aphasia. Brain, 136(6), 1901-1912. Retrieved from http://dx.doi.org/10.1093/brain/awt087

Thompson, H. E., Robson, H., Lambon Ralph, M. A., \& Jefferies, E. (2015). Varieties of semantic 'access' deficit in Wernicke's aphasia and semantic aphasia. Brain, 138(12), 3776-3792. Retrieved from http://dx.doi.org/10.1093/brain/awv281

Tremblay, K., Kraus, N., McGee, T., Ponton, C., \& Otis, B. (2001). Central auditory plasticity: changes in the N1-P2 complex after speech-sound training. Ear and Hearing, 22(2), 79-90. http://doi.org/10.1097/00003446-200104000-00001

Van de Steen, F., Faes, L., Karahan, E., Songsiri, J., Valdes-Sosa, P. A., \& Marinazzo, D. (2016). Critical comments on EEG sensor space dynamical connectivity analysis. Brain Topography, $1-12$.

Weisz, N., \& Obleser, J. (2014). Synchronisation signatures in the listening brain: a perspective from non-invasive neuroelectrophysiology. Hearing Research, 307, 16-28. 
http://doi.org/10.1016/j.heares.2013.07.009 